\title{
Holographic entanglement entropy close to quantum phase transitions
}

\author{
Yi Ling, ${ }^{a, b}$ Peng Liu, ${ }^{a}$ Chao Niu, ${ }^{a}$ Jian-Pin $\mathbf{W u}^{c, b}$ and Zhuo-Yu Xian ${ }^{a}$ \\ ${ }^{a}$ Institute of High Energy Physics, Chinese Academy of Sciences, \\ Beijing 100049, China \\ ${ }^{b}$ State Key Laboratory of Theoretical Physics, Institute of Theoretical Physics, \\ Chinese Academy of Sciences, Beijing 100190, China \\ ${ }^{c}$ Institute of Gravitation and Cosmology, Department of Physics, \\ School of Mathematics and Physics, Bohai University, Jinzhou 121013, China \\ E-mail: lingy@ihep.ac.cn, liup51@ihep.ac.cn, niuc@ihep.ac.cn, \\ jianpinwu@gmail.com, xianzy@ihep.ac.cn
}

ABSTRACT: We investigate the holographic entanglement entropy (HEE) of a strip geometry in four dimensional Q-lattice backgrounds, which exhibit metal-insulator transitions in the dual field theory. Remarkably, we find that the HEE always displays a peak in the vicinity of the quantum critical points. Our model provides the first direct evidence that the HEE can be used to characterize the quantum phase transition (QPT). We also conjecture that the maximization behavior of HEE at quantum critical points would be universal in general holographic models.

KEYwORDS: Holography and condensed matter physics (AdS/CMT), Gauge-gravity correspondence

ArXiv EPrint: 1502.03661 


\section{Contents}

1 Introduction 1

2 The holographic setup and phase diagram 1

3 HEE and numerical results 3

4 Discussion $\quad 6$

\section{Introduction}

Quantum phase transitions (QPT) are believed to give rise to some of the most interesting phenomena in condensed matter physics [1]. Yet a systematic characterization of QPTs remains an outstanding open question. Entanglement could provide an important handle for understanding QPT. For example, there have been indications that entanglement is enhanced at a quantum critical point (QCP) [2-5](for a review, see [6]). Furthermore, change of entanglement patterns may underlie certain QPTs which do not involve symmetry breaking or traditional order parameters.

QPTs are often very difficult to analyze as they naturally occur in strongly correlated many-body systems. Entanglement entropy is also notoriously hard to calculate. The AdS/CFT correspondence provides powerful tools for both analyzing QPTs and computing entanglement entropy $[7,8]$ in strongly coupled systems.

In this paper we show that in a class of strongly coupled holographic systems undergoing QPTs, entanglement entropy attains a maximum at the corresponding QCPs. Thus entanglement entropy can be used as a diagnostic for QPT. Behavior of entanglement entropy near various holographic thermal phase transitions were investigated before in $[9-12]$.

\section{The holographic setup and phase diagram}

Metal-insulator transitions are observed in many condensed matter systems and often involve strongly coupled physics. Recently there has been progress in simulating metalinsulator transitions in holographic systems in (2+1)-dimension [13-16]. Recall that the radial direction of an asymptotically Anti de-Sitter (AdS) spacetime can be understood as a geometrization of the renormalization group (RG) flow for the dual field theory. The IR physics is then encoded in the geometry of the deep interior of the spacetime, to which we will refer to as the IR geometry. Thus a metal-insulator transition is realized on the gravity side as a geometric transition: as external parameter(s) are dialed, the IR geometry deforms from that describing a metallic phase to that corresponding to an insulator. At a 
finite chemical potential, the IR geometry describing a metallic phase is well known, given by $A d S_{2} \times R^{2}$ [17]. The key to recent development came from construction of the so-called Q-lattice models [14] which give rise to new IR geometries dual to insulators.

Let us now describe the gravity set up for Q-lattice models. The Lagrangian of the gravity dual can be written as

$$
\mathcal{L}=R+6-\frac{1}{2} F^{\mu \nu} F_{\mu \nu}-|\nabla \Phi|^{2}-m^{2}|\Phi|^{2}
$$

where the AdS length scale is set to unity. $\Phi$ is a complex field which will be used to introduce a lattice structure along one of the spatial directions, say $x$, by the ansatz $\Phi=e^{i \tilde{k} x} \varphi$ with $\varphi x$-independent. A remarkable feature of such an ansatz is that while translational symmetry is broken, the gravity equations of motion are still ODEs instead of PDEs, bringing great simplifications. This construction is analogous to the construction of Qballs which is employed to build spherically symmetric solitons in [18], thus is called the holographic Q-lattices.

The full solution to equations of motion of (2.1) can be written in a form

$$
\begin{aligned}
& d s^{2}=\frac{1}{z^{2}}\left(-P(z) d t^{2}+\frac{d z^{2}}{P(z)}+V_{1} d x^{2}+V_{2} d y^{2}\right), \\
& A=\mu(1-z) a d t, \quad \Phi=e^{i \tilde{k} x} z^{3-\Delta} \phi,
\end{aligned}
$$

where $P(z) \equiv U(1-z)\left(1+z+z^{2}-\mu^{2} z^{3} / 2\right)$ and $\Delta=3 / 2 \pm\left(9 / 4+m^{2}\right)^{1 / 2}$. Notice that $U, V_{1}, V_{2}, a$ and $\phi$ are functions of the radial coordinate $z$ only and $\mu$ is the chemical potential of the dual field theory. We set the boundary condition for $\phi$ as $\phi(0) \equiv \tilde{\lambda}$, which is understood as the lattice amplitude. Then the solutions of the background are specified by three dimensionless parameters, namely the temperature $\tilde{T} / \mu$, lattice amplitude $\tilde{\lambda} / \mu^{3-\Delta}$, and lattice wave number $\tilde{k} / \mu$. For simplicity, we will denote these quantities in short by $T, \lambda, k$ through this paper. The metric has an event horizon at $z=1$ and the spacetime boundary is at $z=0$. The Hawking temperature is $T=\left(6-\mu^{2}\right) U(1) /(8 \pi \mu)$. We will work at temperature $T=0.001$, which is low enough to observe quantum critical phenomenon. In addition, we will set the mass of the scalar field as $m^{2}=-2$, with a brief discussion on other values of the mass in the end of this paper.

The phase diagram for the metal-insulator transition over Q-lattice backgrounds (2.2) can be obtained by examining the DC conductivity $\sigma_{\mathrm{DC}}$ of the dual field theory along $x$ direction, and is presented in figure 1. $\sigma_{\mathrm{DC}}$ can be computed using standard methods and it can be written in terms of various geometric quantities in (2.2) evaluated at the horizon, i.e.

$$
\sigma_{\mathrm{DC}}=\left.\left(\sqrt{\frac{V_{2}}{V_{1}}}+\frac{\mu^{2} a^{2} \sqrt{V_{1} V_{2}}}{k^{2} \phi^{2}}\right)\right|_{z=1} .
$$

As a practical diagnostic, we classify the dual system into a metallic phase or an insulating phase by the temperature dependence of DC conductivity around $T=0.001$. More explicitly, for the metallic phase we expect $\partial_{T} \sigma_{\mathrm{DC}}<0$, while for the insulating phase $\partial_{T} \sigma_{\mathrm{DC}}>0$. As a result, the QCPs are characterized by $\partial_{T} \sigma_{\mathrm{DC}}=0$. They form a critical line in $k-\lambda$ plane as shown in figure 1 . We stress that this phase structure has little change when decreasing the temperature further. 


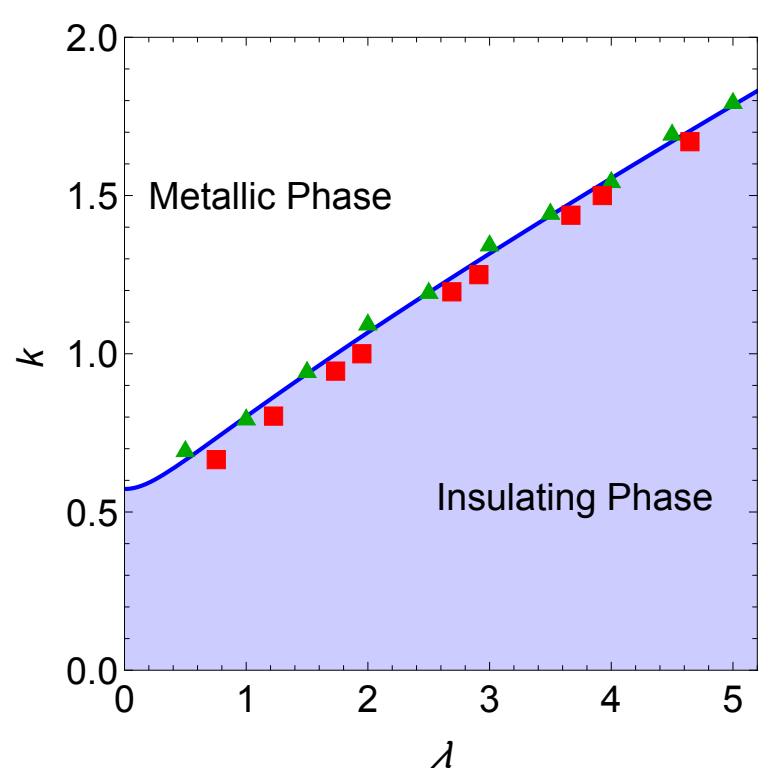

Figure 1. The phase diagram over $(\lambda, k)$ plane $(\lambda \neq 0, k \neq 0)$ for the metal-insulator transition in Q-lattice background. The upper and the lower region of the plot corresponds to metallic and insulating phase, respectively. The HEE with large $l$ is numerically computed through eq. (3.3). Green triangles represent the peaks of HEE when varying $k$ but fixing $\lambda$, while red squares are peaks of HEE when varying $\lambda$ but fixing $k$.

\section{HEE and numerical results}

In the AdS/CFT correspondence the entanglement entropy for a region $A$ is obtained from gravity as the area of the minimal surface $\gamma_{A}$ in the bulk geometry which ends at $\partial A$ [7], i.e.

$$
S_{A}=\frac{\operatorname{Area}\left(\gamma_{A}\right)}{4 G_{N}}
$$

where $G_{N}$ is the bulk Newton constant.

We now consider the holographic entanglement entropy (HEE) for a strip region in the boundary system described by (2.2). We take strip to stretch in $y$-direction with length $L_{y}(\rightarrow \infty)$ and $x$-direction with width $2 l \ll L_{y}$. While the translational symmetry along $x$ direction is broken by $\Phi$, the metric components in (2.2) are still functions of $z$. It is then straightforward to find the minimal surface for the strip region, which can be specified by the location $z_{*}$ of the bottom of the minimal surface in $z$-direction. We find that $z_{*}$ satisfies the equation

$$
l=\mu \int_{0}^{z_{*}} d z z^{2} \sqrt{\frac{V_{1}\left(z_{*}\right) V_{2}\left(z_{*}\right)}{P(z) V_{1}(z) W\left(z_{*}, z\right)}}
$$

where $W\left(z_{*}, z\right) \equiv z_{*}^{4} V_{1}(z) V_{2}(z)-z^{4} V_{1}\left(z_{*}\right) V_{2}\left(z_{*}\right)$.

From eq. (3.1) we find that after subtracting the vacuum part the entanglement entropy $S$ behaves as

$$
S=\frac{L_{y}}{2 \mu G_{N}}\left\{-\frac{1}{z_{*}}+\int_{0}^{z_{*}} \frac{d z}{z^{2}}\left[\frac{z_{*}^{2} V_{1}(z) V_{2}(z)}{\sqrt{P(z) V_{1}(z) W\left(z_{*}, z\right)}}-1\right]\right\}
$$



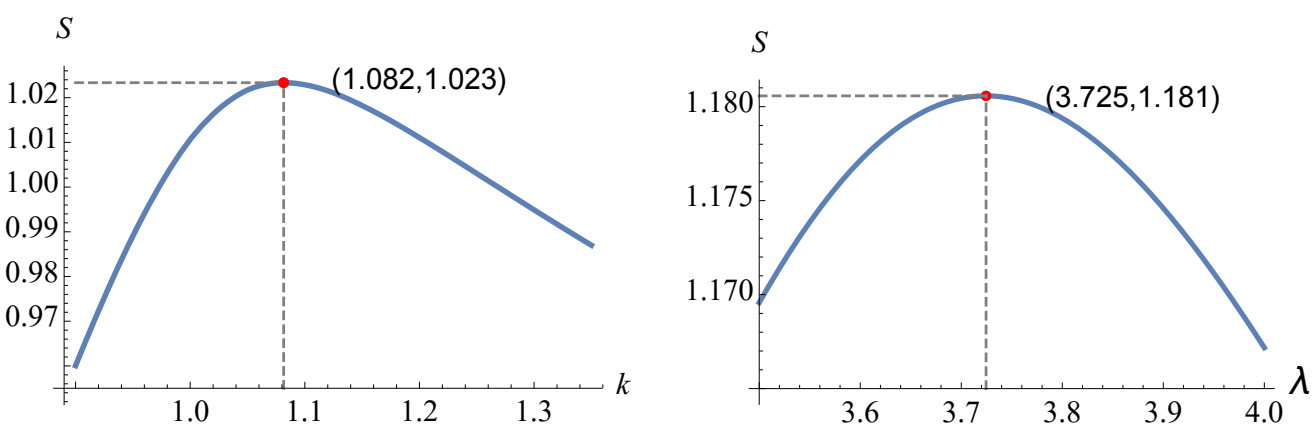

Figure 2. The left plot is for HEE $S$ as a function of the lattice wavenumber $k$ with $l=5.56, \lambda=2$, while the right plot is for $S$ as a function of the lattice amplitude $\lambda$ with $l=5.44, k=1.44$.

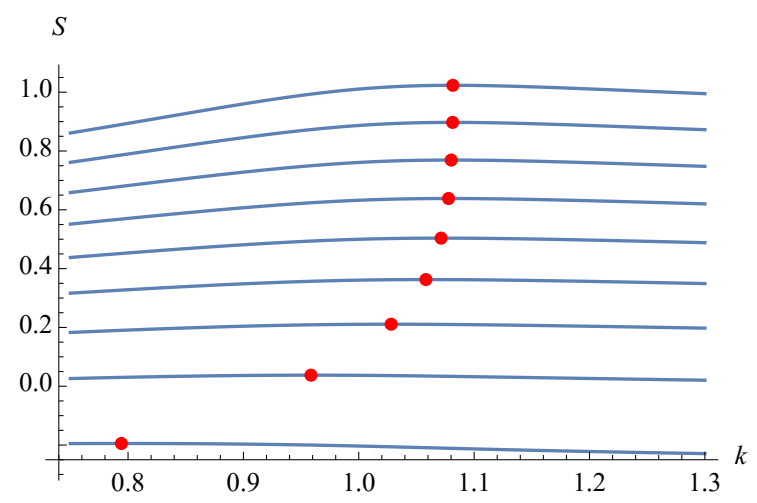

Figure 3. The parameter $\lambda$ is fixed at $\lambda=2$ for the above plot. The turning point approaches the critical point when the width of the strip is increased from 0.91 to 5.56 uniformly. The maximum of each curve is marked as a red dot in figure.

Firstly, we compute the HEE $S$ as a function of the parameter $k$ and $\lambda$ separately when the strip width $2 l$ is finite but fixed at a relatively large value. Our results are illustrated in figure 2. Interestingly enough, we observe that the HEE displays a pronounced peak in both plots. Moreover, we find that the location of such turning points is independent of the width of the strip when $l$ is relatively large. To show this we plot the shift of the peaks of HEE with the width of the strip in figure 3. Evidently, the peaks of the HEE converge to a fixed point with a definite value of $k$ when the width of the strip is becoming large enough. The same convergent behavior is also observed in the case when varying the parameter $\lambda$ with $k$ fixed. Secondly, we mark the turning points of the HEE with large $l$ in the $(\lambda, k)$ plane of the phase diagram as illustrated in figure 1. Remarkably, we observe that all the turning points of the HEE are distributed in the vicinity of the trajectory of the critical points, clearly indicating that HEE can be used to characterize the occurrence of QPTs.

Next, we are interested in the large distance behavior, i.e. $l \rightarrow \infty$. In this limit it can be readily seen from (3.2) that $z_{*} \rightarrow 1$, i.e. the bottom of the minimal surface approaches the horizon. It then follows from (3.3) that $S$ is dominated by the contribution of the part of the minimal surface near the horizon. More explicitly, as $z_{*} \rightarrow 1$ from (3.2) and (3.3), 
both $S$ and $l$ are logarithmic divergent,

$$
\begin{aligned}
S & \left.\approx \frac{V_{2} L_{y}}{2 \mu G_{N}} \sqrt{\frac{V_{1}}{p U B}}\right|_{z=1} \log \left(\frac{1}{1-z_{*}}\right)+\cdots, \\
l & \left.\approx \mu \sqrt{\frac{V_{2}}{p U B}}\right|_{z=1} \log \left(\frac{1}{1-z_{*}}\right)+\cdots,
\end{aligned}
$$

where $B \equiv 4 V_{1} V_{2}-V_{1}^{\prime} V_{2}-V_{1} V_{2}^{\prime}$ and $p=1+z+z^{2}-\mu^{2} z^{3} / 2$. Thus for large $l$,

$$
S=r V_{\text {strip }}+\cdots, \quad \text { with } \quad r=\left.\frac{\sqrt{V_{1} V_{2}}}{2 G_{N} \mu^{2}}\right|_{z=1},
$$

is proportional to the volume $V_{\text {strip }}=l L_{y}$ of the strip, with $r$ the entropy density. Note that $S$ is given by the horizon area and thus coincides with the black hole entropy. Thus in the large distance limit, $S$ is solely determined by the horizon geometry, which is of course expected. Note that the result is in fact general, applying to generic gravity geometry and entangling region of any shape (not just strip).

In figure 4 we give the contour plot of $r$ over the $(\lambda, k)$-plane. Again, we see the $r$ achieves local maxima precisely at the critical line identified from the DC conductivity earlier. In particular, we show an example in this figure to locate the maximal value of $r$ along the vertical direction (a short green line) and the horizontal direction (a short red line), which are marked by a green dot and a red dot, respectively. In general we find the data in this method matches rather closely with figure 1 . In other words, in long distance limit entanglement entropy can also be used as a diagnostic for the critical line of QPTs. Moreover, in this contour plot we observe that the peaks of HEE form a ridge along the direction of the critical line. Thus, when we change a single parameter to locate the position of HEE peaks over two or higher dimensional phase diagram, the result obviously depends on the direction of observation (or the direction of cutting ridge). Unlike in our current paper, in general cases the local HEE peaks obtained in this manner may deviate far from critical points in certain directions. Therefore, to avoid this confusion, we may present a more strict statement on the relation between HEE and QPTs for a multi-parameter system. That is, the peaks of HEE always form a ridge along the critical line, and a pronounced peak can be observed at the critical point when computing HEE along the direction perpendicular to the critical line over the phase diagram.

Our result for holographic systems resonates well with earlier observations [2-5] in the condensed matter literature regarding entanglement entropy and QCPs.

Furthermore, we have investigated the behavior of $r$ at lower temperatures. We find that with the decrease of temperature, the location of the maximum over the phase space tends to fix. In metallic phase with fixed parameters the quantity $r$ converges to non-zero values. While in insulating phase $r$ tends to be vanishing in zero temperature limit, which implies the violation of the volume law of HEE. As a result, the peaks of HEE become steep on the insulating side with the decease of the temperature. This phenomenon suggests that the maximization behavior of HEE at finite temperature might be the reflection of the discontinuity of HEE at zero temperature. However, as disclosed in [13], in this case the 


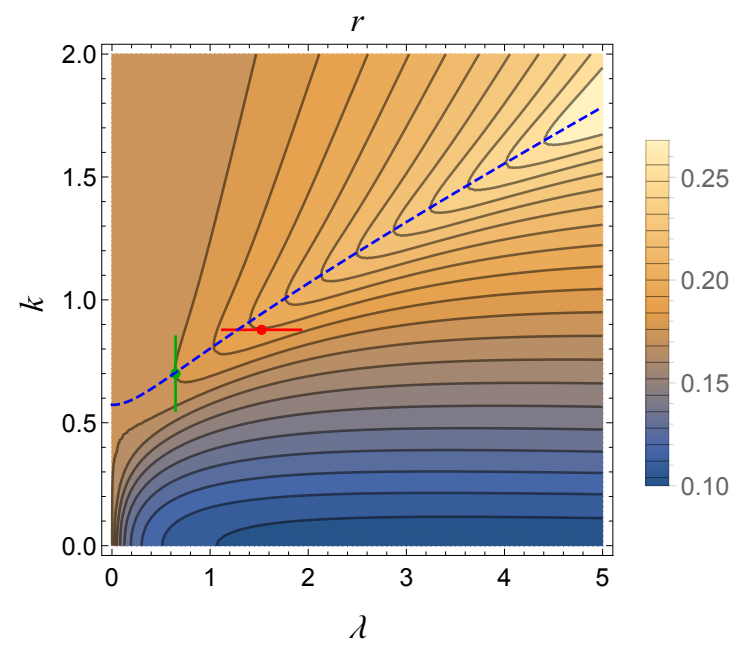

Figure 4. A contour plot of the $r$, whose values are represented by different colors. Each grey trajectory has the same value of $r$. The blue dashed curve is the critical line where metal-insulator transition occurs.

quantum phase transition could still be continuous and infinite order since the free energy in the bulk does not exhibit any discontinuous behavior even at zero temperature. Finally, it is worthwhile to point out that for insulating phases there could exist a kink $\left(r^{\prime}\left(T_{c}\right)=0\right)$ at $T_{c} \simeq 10^{-5}$ as described in [14]. However, for $T<T_{c}$ the thermodynamically preferred solution still exhibits insulating behavior and $r$ has the singular behavior $\lim _{T \rightarrow 0} r \rightarrow$ 0 . Therefore for even lower temperature, the phase structure illustrated in figure 1 will be stable.

We conjecture that the discontinuity of HEE at zero temperature may result from the artifact of IR geometry $A d S_{2}$. As pointed out in [13, 19], the IR geometry $A d S_{2}$ will lead to the violation of the third law of thermodynamics due to the non-vanishing entropy density at zero temperature, and may therefore be an artifact of large $N$ limit of holography. Nevertheless, we intend to argue that the maximization behavior of HEE disclosed in our paper may not suffer from this artifact. In addition to the near horizon limit analysis shown above, figure 3 shows that HEE with finite $l$ with $l T \ll 1$ also exhibits maximal behavior at critical points, which is not dictated by the near horizon geometry since the minimal surface can be distant from the horizon. This phenomenon can be understood as that the HEE with finite $l$ actually captures the quantum phase structure at the energy level specified by $z_{*}$, namely the bottom location of the minimal surface. Furthermore, the peaks of HEE converge to fixed values near the QCP when $l$ becomes large (see figure 1). Therefore, in spite of the subtlety of IR geometry $A d S_{2}$, we believe the maximization behavior of HEE at QPTs should be genuine and universal in the holographic approach.

\section{Discussion}

Throughout this paper we have worked with a scalar mass $m^{2}=-2$ because in this case we find the numerical analysis exhibits convergent behavior very well. Nevertheless, it is worth 
to point out that in this setup the $A d S_{2} B F$ bound is violated such that new phases could be found at lower temperatures [14]. Taking this into account, we have also performed the calculation for the mass $m^{2}=-3 / 2$ which does not violate $A d S_{2} B F$ bound. We qualitatively obtain the same behavior of the HEE, but with much higher precision and more grid points.

As a summary, in this paper we have computed the HEE of a strip geometry in four dimensional Q-lattice background, which exhibits metal-insulator transitions in the dual field theory. We have found that the behavior of the HEE in long distance physics is dominantly determined by the near horizon geometry of Q-lattice. More importantly, through numerical calculation we have demonstrated that the HEE attains a maximum at the corresponding QCPs. This fact reveals that HEE can be used as a diagnostic for QPTs indeed. Based on what we have observed in Q-lattice setup, we have following remarks and conjectures which should be crucial for next investigations on QPT in holographic approach. Firstly, we conjecture that the connection between HEE and QPT which is captured by the near horizon geometry would be a universal feature for general holographic models. Secondly, we further propose a simple but elegant criteria for the occurrence of QPT in holographic approach, that would be the existence of a maximum for the area element of black hole horizon when changing the parameters of the system in zero temperature limit. Finally, it is crucial to explore the scaling behavior of the HEE around QCPs in the zero temperature limit, which should be done in a background which contains IR geometry dual to metallic phases with vanishing entropy density, rather than $A d S_{2}$ with finite entropy density. Our investigation on these topics is under progress.

\section{Acknowledgments}

We express our special thanks to Hong Liu for dramatically improving the manuscript. We are also grateful to Xiaomei Kuang, Weijia Li, Rene Meyer, Philip Phillips, Yu Tian, Yidun Wan, Kun Yang, Hongbao Zhang for stimulating discussion and helpful correspondence. This work is supported by the Natural Science Foundation of China under Grant Nos.11275208, 11305018 and 11178002 . Y.L. also acknowledges the support from Jiangxi young scientists (JingGang Star) program and 555 talent project of Jiangxi Province. J. P. Wu is also supported by Program for Liaoning Excellent Talents in University (No. LJQ2014123).

Open Access. This article is distributed under the terms of the Creative Commons Attribution License (CC-BY 4.0), which permits any use, distribution and reproduction in any medium, provided the original author(s) and source are credited.

\section{References}

[1] S. Sachdev, Quantum phase transitions, Cambridge University Press, Cambridge U.K. (2000).

[2] A. Osterloh, L. Amico, G. Falci and R. Fazio, Scaling of entanglement close to a quantum phase transitions, Nature 416 (2002) 608 [quant-ph/0202029]. 
[3] T.J. Osborne and M.A. Nielsen, Entanglement in a simple quantum phase transition, Phys. Rev. A 66 (2002) 032110 [INSPIRE].

[4] G. Vidal, J.I. Latorre, E. Rico and A. Kitaev, Entanglement in quantum critical phenomena, Phys. Rev. Lett. 90 (2003) 227902 [quant-ph/0211074] [INSPIRE].

[5] Y. Chen, P. Zanardi, Z.D. Wang and F.C. Zhang, Sublattice entanglement and quantum phase transitions in antiferromagnetic spin chains, New J. Phys. 8 (2006) 97.

[6] L. Amico, R. Fazio, A. Osterloh and V. Vedral, Entanglement in many-body systems, Rev. Mod. Phys. 80 (2008) 517 [quant-ph/0703044] [INSPIRE].

[7] S. Ryu and T. Takayanagi, Holographic derivation of entanglement entropy from AdS/CFT, Phys. Rev. Lett. 96 (2006) 181602 [hep-th/0603001] [INSPIRE].

[8] T. Takayanagi, Entanglement entropy from a holographic viewpoint, Class. Quant. Grav. 29 (2012) 153001 [arXiv:1204.2450] [INSPIRE].

[9] T. Albash and C.V. Johnson, Holographic studies of entanglement entropy in superconductors, JHEP 05 (2012) 079 [arXiv: 1202.2605] [INSPIRE].

[10] R.-G. Cai, S. He, L. Li and Y.-L. Zhang, Holographic entanglement entropy in insulator/superconductor transition, JHEP 07 (2012) 088 [arXiv: 1203.6620] [INSPIRE].

[11] R.E. Arias and I.S. Landea, Backreacting p-wave superconductors, JHEP 01 (2013) 157 [arXiv: 1210.6823] [INSPIRE].

[12] X.-M. Kuang, E. Papantonopoulos and B. Wang, Entanglement entropy as a probe of the proximity effect in holographic superconductors, JHEP 05 (2014) 130 [arXiv:1401.5720] [INSPIRE].

[13] A. Donos and S.A. Hartnoll, Interaction-driven localization in holography, Nature Phys. 9 (2013) 649 [arXiv: 1212.2998] [INSPIRE].

[14] A. Donos and J.P. Gauntlett, Holographic Q-lattices, JHEP 04 (2014) 040 [arXiv: 1311.3292] [INSPIRE].

[15] A. Donos and J.P. Gauntlett, Novel metals and insulators from holography, JHEP 06 (2014) 007 [arXiv: 1401.5077] [INSPIRE].

[16] Y. Ling, C. Niu, J. Wu, Z. Xian and H.-b. Zhang, Metal-insulator transition by holographic charge density waves, Phys. Rev. Lett. 113 (2014) 091602 [arXiv: 1404.0777] [INSPIRE].

[17] S.A. Hartnoll and D.M. Hofman, Locally critical resistivities from Umklapp scattering, Phys. Rev. Lett. 108 (2012) 241601 [arXiv:1201.3917] [INSPIRE].

[18] S.R. Coleman, Q balls, Nucl. Phys. B 262 (1985) 263 [Erratum ibid. B 269 (1986) 744] [INSPIRE].

[19] N. Iqbal, H. Liu and M. Mezei, Lectures on holographic non-Fermi liquids and quantum phase transitions, arXiv:1110.3814 [INSPIRE]. 